\title{
Imaging-Histological Discordance after Sonographically Guided Percutaneous Breast Core Biopsy
}

\author{
Aykut Soyder $^{\mathrm{a}}$ Füsun Taşkın ${ }^{\mathrm{b}}$ Serdar Ozbas $^{\mathrm{c}}$ \\ ${ }^{a}$ Department of General Surgery, Adnan Menderes University Faculty of Medicine, Aydin, Turkey; \\ ${ }^{b}$ Department of Radiology, Adnan Menderes University Faculty of Medicine, Aydin, Turkey; \\ c Guven Hospital, Ankara, Turkey
}

\section{Keywords}

Imaging-histological discordance - Breast cancer . Core biopsy

\section{Summary}

Background: The objectives of this study were to determine the frequency of imaging-histological discordance and to compare the frequency of carcinoma between discordant lesions at ultrasound (US)-guided core needle biopsy. Materials and Methods: From November 2009 to June 2012, we performed US-guided 14-gauge core needle biopsies on 989 breast lesions in 961 women. We reviewed $58(5.8 \%)$ cases that had imaging-histological discordance after percutaneous breast biopsy and underwent subsequent excisional biopsy. The clinical, radiological, and histological findings were reviewed for those 58 cases. Results: Among the 58 cases, subsequent excisions revealed $16(27.5 \%)$ malignancies, which were categorized as $9(15.5 \%)$ invasive ductal carcinomas, 4 (6.9\%) malignant phyllodes tumors, and 3 (5.1\%) ductal carcinomas in situ. Conclusion: The malignancy rate of $27.5 \%$ suggests that surgical excision should be performed in those cases presenting with imaging-histological discordance after US-guided core biopsy. Careful correlation of clinical, radiological, and histological results as well as appropriate follow-up are essential.

We have submitted a short version of this work as a poster presentation during the 2012 European Society of Surgery (ESS) Congress in Istanbul/Turkey.

\section{Introduction}

Imaging and pathological findings are considered to be concordant when the pathological results provide an acceptable explanation for the imaging features, and discordant when they do not [1]. Once the assessment for concordance is completed, a management plan can be provided. Parikh and Tickman [2] described 5 possible outcomes of imaging-pathology correlation and suggested corresponding management for each category. 1 of them, 'discordant benign lesion', is suspicious for malignancy at imaging (i.e., Breast Imaging Reporting and Data System (BI-RADS) category 4 or 5) but demonstrates imaging-histological discordance at percutaneous breast biopsy and gives benign pathological results following core needle biopsy (CNB) [3]. Benign lesions with speculative findings can simulate malignant lesions and may be considered in the differential diagnosis as the following: sclerosing adenosis, fat necrosis, mastitis, diabetic mastopathy, fibrocystic changes, ruptured inflammatory cysts, granulomatous mastitis, fibroadenomas, fibroadenomatous mastopathy, and apocrine metaplasia [4]. However, the radiologist should pay special attention to discordant benign lesions a substantial number of which are cancers missed at CNB. In published reports, up to $64 \%$ of discordant lesions after percutaneous biopsy were confirmed as cancer by subsequent surgical excision [5]. For an ultrasound (US)-guided 14 -gauge $\mathrm{CNB}$, discordant lesions had cancer rates of up to $50 \%$ [6]. If there is concern regarding a discordant benign lesion, it is prudent for the radiologist to immediately contact and thoroughly communicate with the interpreting pathologist. According to the outcome of the discussion, the radiologist should report the result and discuss the need for a repeat biopsy with the referring physician or the patient. A surgical biopsy, rather than a CNB, is recommended for a repeat biopsy because of the inconclusive outcome of the first CNB. In recent studies that have assessed discordance

\section{KARGER \\ Fax +497614520714

\section{(c) 2015 S. Karger GmbH, Freiburg}

$1661-3791 / 15 / 0101-0033 \$ 39.50 / 0$
Aykut Soyder, MD 
rates with stereotactic and US-guided CNB, imaging-histological discordance was reported in 3\% (range 1-8\%) of all lesions; among discordant lesions in those studies, the frequency of cancer at re-biopsy was $14 \%$ (range $0-100 \%$ ) [1, 7-10]. Imaging-histological discordance at stereotactic or US-guided CNB is an indication for re-biopsy, usually surgical excision, to exclude the presence of carcinoma [1]. The current study was undertaken to determine the frequency of discordance at US-guided 14-gauge CNB for category 4 or 5 lesions, and to assess the cancer rate at excisional biopsy in discordant lesions.

\section{Materials and Methods}

Our study is a retrospective review of data obtained from the clinical, radiology, and pathology archives of our institution. An application was not submitted to the ethics committee.

\section{Biopsy Technique}

Between November 2009 and June 2012, percutaneous US-guided CNB was performed on 989 consecutive breast lesions in 961 women that were prospectively classified according to BI-RADS as category 4 (suspicious) or category 5 (highly suggestive of malignancy) at our institution. Freehand technique guided by a 7.5-MHz linear transducer mounted on an EUB 420 US unit (Hitachi Medical Systems, Tokyo, Japan) and with a 6- to $12-\mathrm{MHz}$ broadband transducer mounted on an Acuson Antares US unit (Siemens, Mountain View, CA, USA) was used in each procedure. All procedures were performed with an automated biopsy gun (Bard Magnum, Covington, Georgia, USA) and a 14-gauge core needle with a 22 -mm throw, and 4 (minimum 3, maximum 8) core samples were usually obtained. More or fewer core samples were occasionally obtained at the discretion of the radiologist. Informed consent was obtained from all patients undergoing percutaneous biopsy procedures.

\section{Imaging-Histological Correlation: Definition of Discordance}

Imaging and histological findings were considered 'discordant' when the histological findings did not provide an acceptable explanation for the imaging features. Discordant lesions included lesions that were suspicious for malignancy, lesions in which the histological findings did not account for the imaging pattern, and lesions that were highly suggestive of malignancy but were sampled with benign results, unless the specific histology accounted for the imaging features.

\section{Wire-Guided Procedure}

Lesions that could be detected by either mammography or US were marked with the help of whichever method applied. Lesions that could be detected by both mammography and US were marked with the help of US. Spring-hook type guide wire was used in the localization procedure. The procedure was performed under local anesthesia. The guide wire was placed into the lesion through the shortest way using anteroposterior orientation, and the marking was considered to be successful if the leading end of the wire was in or adjacent to (up to $1 \mathrm{~cm}$ ) the lesion. The area marked by the wire was removed along with intact surrounding tissue of at least $1 \mathrm{~cm}$ via surgical excision under general anesthesia. The contours of the specimen were marked with sutures to provide a guide for the pathology department. To find out whether the marked lesions were totally excised or not, specimen radiography was requested for all lesions regardless of their radiological features.

Finally, our study population consisted of 58 patients showing discordance between benign histological findings of a CNB and suspicious imaging findings. 58 cases underwent subsequent wire-guided surgical excision. We reviewed the clinical findings and histopathological results of $\mathrm{CNB}$ and surgical excision of the 58 discordant cases.

\section{Statistical Analysis}

Concordance of the quantitative data with the normal distribution was examined using the Kolmogorov-Smirnov test. In the analysis of the data concordant with the normal distribution, t-test was used for the independent groups, and the definitive statistics were shown as mean \pm standard deviation. In the analysis of the data discordant with the normal distribution, the MannWhitney $U$ test was used, and the definitive statistics were shown as median (25-75 percentile). The chi-square test was used to analyze the qualitative data. $\mathrm{p}<0.05$ was considered statistically significant.

\section{Results}

We performed US-guided 14-gauge CNB on 989 breast lesions in 961 women. We reviewed 58 (5.8\%) cases that had imaging-histological discordance after percutaneous breast biopsy and underwent subsequent excisional biopsy. The clinical findings and pathological results of US-guided 14-gauge CNB are summarized in table 1. Of the 58 discordant cases, the pathological results for surgical excision were malignant in $16(27.5 \%)$ cases. All 58 patients were women (mean age 50.4 years). Between benign $(n=42)$ and malignant ( $\mathrm{n}=16$, upgrade group) cases, no statistically significant differences were found ( $p>0.05$ ) in terms of age, age at menarche, age at birth of first child, number of children, history of breast surgery (for benign lesions), oral contraceptive use, and breast feeding duration.

Family history of breast cancer (1 first-degree relative) was present in the overall discordant group $(\mathrm{n}=58)$ in $15(25.9 \%)$ cases, and specifically in the upgrade group $(n=16)$ in $5(31.3 \%)$ cases. With respect to tumor location in the discordant group, in 34 (58.6\%) cases the primary tumor was located in the upper outer quadrant, in $8(13.8 \%)$ cases in the upper inner quadrant, in 14 $(24.1 \%)$ cases in the retro-areolar region (central), in $1(1.7 \%)$ case in the lower outer quadrant, and in $1(1.7 \%)$ case in the lower inner quadrant. In the upgrade group, in $9(56.3 \%)$ cases the primary tumor was located in the upper outer quadrant, in 3 (18.8\%) cases in the upper inner quadrant, and $4(25 \%)$ cases in the retro-areolar region (central).

Of a total of 58 discordant lesions, 45 (77.5\%) were BI-RADS category 4 , and 13 (22.5\%) were BI-RADS category 5 . Of the 45 lesions that were classified as BI-RADS 4, $36(80 \%)$ were diagnosed as benign and $9(20 \%)$ were diagnosed as malignant. Of the 13 lesions that were classified as BI-RADS 5, 6 (46\%) were benign and 7 (44\%) were malignant. The malignancy rate was higher for lesions that were BI-RADS 5 compared to BI-RADS 4 (7/13, 53.8\%; 9/45, $20 \%)(\mathrm{p}=0.021)$.

For the upgrade group $(n=16)$, the histopathological results of the initial US-guided 14-gauge CNB were as follows: 3 (18.7\%) cases of benign breast tissue, 4 (25\%) cases of focal fibrosis (fig. 1), 4 (25\%) cases of ductal hyperplasia, 2 (12.5\%) cases each of fibroadenoma and benign papilloma, and 1 (6.3\%) case of sclerosing adenosis. Subsequent excision revealed the following malignancies: 9 (56.3\%) invasive ductal carcinomas (figs. 2 and 3), 4 (25\%) phyllodes tumors, and 3 (18.8\%) ductal carcinomas in situ. 7 (43.8\%) cases in the upgrade group presented with a palpable lump (table 2). 
Fig. 1. A 64-year-old woman was referred to the breast imaging unit for suspicious bone lesions. Her left breast was completely fatty on mammography (not seen here). a Mammography showed local asymmetrically increased density in the upper outer quadrant of the right breast. b, c Transverse sonograms showed 3 irregular-shaped, hypoechoic lesions with ill-defined margins in the same location in the right breast. Ultrasound (US)-guided 14gauge core needle biopsy yielded a histopathological diagnosis of fibrosis. Because of the clinical-radio-
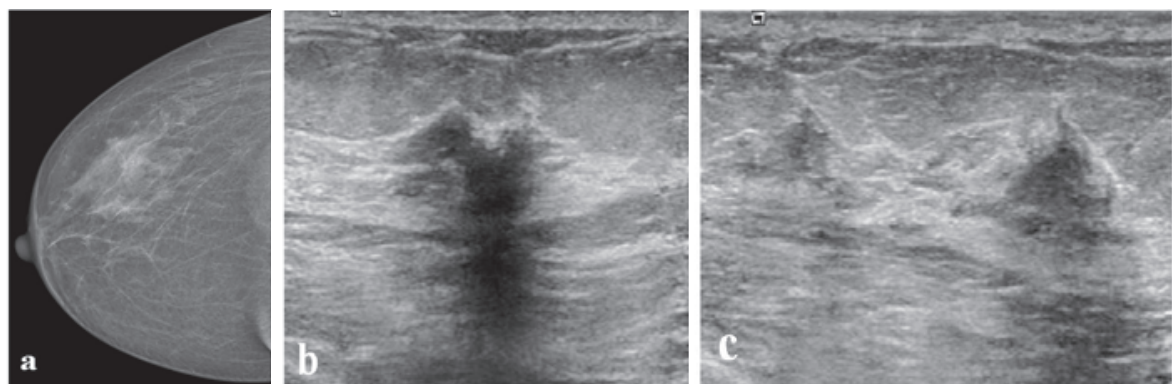

logical-histopathological discordance, surgical excision was performed with US-guided wire needle localization. The final diagnosis did not change.

Fig. 2. Magnified right craniocaudal mammogram of a 57-year-old women with a palpable lump in the right breast. a Mammogram showing a dense mass with partially obscured margins (black arrows).

b Transverse sonogram showing a lobulated hypoechoic solid mass. c Core needle biopsy performed with a 14-gauge needle yielded the histopathological diagnosis of sclerosing adenosis. Surgical excision was performed with wire needle localization; the transverse sonogram shows the localization needle in the lesion. Final diagnosis was invasive ductal carcinoma.
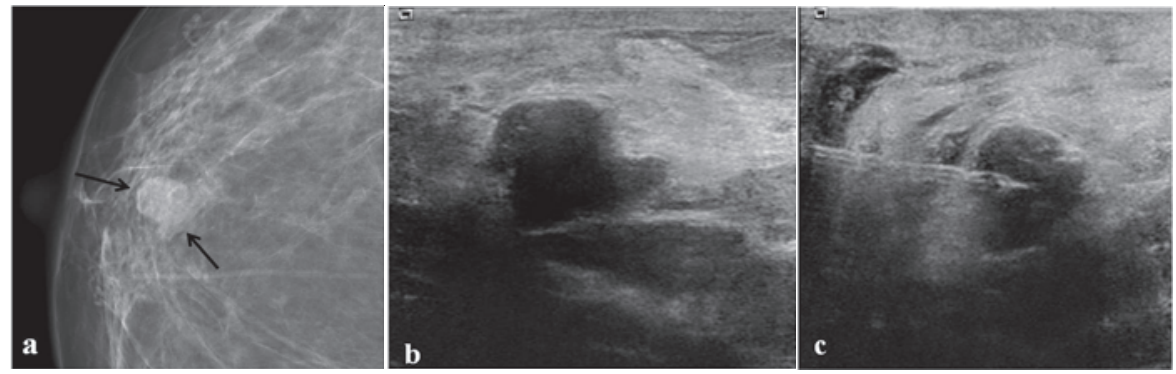

Table 1. Clinical findings and pathological results of the discordant cases, and comparison between benign and malignant cases

\begin{tabular}{|c|c|c|c|c|}
\hline Clinical characteristics & $\begin{array}{l}\text { Total } \\
(\mathrm{n}=58)\end{array}$ & $\begin{array}{l}\text { Benign } \\
(\mathrm{n}=42)\end{array}$ & $\begin{array}{l}\text { Malignant } \\
(\mathrm{n}=16)\end{array}$ & $\mathrm{p}$ \\
\hline Age, years & $50 \pm 11$ & $51 \pm 14$ & $50 \pm 10$ & 0.875 \\
\hline Age at menarche, mean (range), years & $13.3(11-17)$ & $13(12-14)$ & $13.5(12-14)$ & 0.775 \\
\hline $\begin{array}{l}\text { Age at birth of first child, mean (range), } \\
\text { years }\end{array}$ & $22(15-35)$ & $21(18-24)$ & $22(21-26)$ & 0.194 \\
\hline Children, mean (range), $\mathrm{n}$ & $1.9(0-3)$ & $2(2-2.3)$ & $2(1.3-3)$ & 0.595 \\
\hline History of breast surgery, $\mathrm{n}$ & 19 & 12 & 7 & 0.351 \\
\hline Oral contraceptive use, $\mathrm{n}$ & 11 & 7 & 4 & 0.475 \\
\hline Breastfeeding duration, $\mathrm{n}$ & & & & 1.000 \\
\hline $12 \leq$ months & 29 & 21 & 8 & \\
\hline $12>$ months & 29 & 21 & 8 & \\
\hline \multicolumn{5}{|l|}{ Family history of breast cancer, $\mathrm{n}$} \\
\hline 1 first degree relative & 15 & 10 & 5 & \\
\hline No family history & 38 & 27 & 11 & \\
\hline Family history of any cancer & 5 & 5 & 0 & \\
\hline \multicolumn{5}{|l|}{ Tumor location (quadrants),n } \\
\hline Upper outer & 34 & 25 & 9 & \\
\hline Upper inner & 8 & 5 & 3 & \\
\hline Lower outer & 1 & 1 & 0 & \\
\hline Lower inner & 1 & 1 & 0 & \\
\hline Central & 14 & 10 & 4 & \\
\hline BI-RADS category, $\mathrm{n}$ & & & & 0.021 \\
\hline 4 & 45 & 36 & 9 & \\
\hline 5 & 13 & 6 & 7 & \\
\hline \multicolumn{5}{|l|}{ Core needle biopsy results, $\mathrm{n}$} \\
\hline Benign breast tissue & 20 & 17 & 3 & \\
\hline Ductal hyperplasia & 11 & 7 & 4 & \\
\hline Focal fibrosis & 9 & 5 & 4 & \\
\hline Benign papilloma & 6 & 4 & 2 & \\
\hline Fibroadenoma & 5 & 3 & 2 & \\
\hline Sclerosing adenosis & 3 & 2 & 1 & \\
\hline Microglandular adenosis & 2 & 2 & 0 & \\
\hline Apocrine metaplasia & 1 & 1 & 0 & \\
\hline Fibrocystic change & 1 & 1 & 0 & \\
\hline
\end{tabular}


Fig. 3. Magnified left mediolateral oblique mammogram of a 47-year-old asymptomatic woman. a Small dense focus with obscured margins associated with architectural distortion (black arrows). b Transverse sonogram shows hypoechoic solid lesion with ill-defined margins. c Core needle biopsy performed with 14-gauge needle yielded a histopathological diagnosis of ductal hyperplasia and fibrocystic changes. Surgical excision was performed with wire needle localization. The specimen mammogram shows the lesion and localization wire. Final diagnosis was invasive ductal cancer.
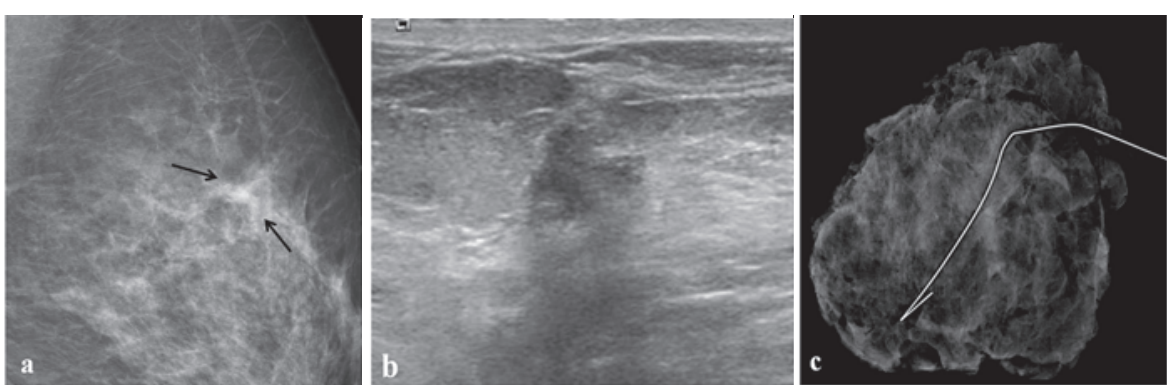

Table 2. Histopathological analysis of the malignant casesa

\begin{tabular}{llll}
\hline $\begin{array}{l}\text { Age, } \\
\text { years }\end{array}$ & Palpability & $\begin{array}{l}\text { Histopathological findings } \\
\text { core biopsy results }\end{array}$ & excisional biopsy results \\
\hline 84 & palpable & focal fibrosis & phyllodes tumor, malignant \\
57 & palpable & benign papilloma & IDC \\
25 & palpable & fibroadenoma & phyllodes tumor, malignant \\
51 & non-palpable & ductal hyperplasia & DCIS \\
55 & non-palpable & benign breast tissue & IDC \\
46 & non-palpable & ductal hyperplasia & IDC \\
59 & palpable & focal fibrosis & DCIS \\
49 & non-palpable & ductal hyperplasia & IDC \\
62 & non-palpable & focal fibrosis & phyllodes tumor, malignant \\
30 & non-palpable & sclerosing adenosis & IDC \\
37 & palpable & benign breast tissue & IDC \\
51 & non-palpable & ductal hyperplasia & IDC \\
38 & palpable & fibroadenoma & phyllodes tumor, malignant \\
62 & non-palpable & benign papilloma & IDC \\
50 & non-palpable & focal fibrosis & DCIS \\
57 & palpable & benign breast tissue & IDC \\
\hline
\end{tabular}

aUpgrade group: discordant cases which had a pathologic diagnosis of malignancy on excisional biopsy.

IDC = Invasive ductal carcinoma; DCIS = ductal carcinoma in situ.

\section{Discussion}

Imaging-guided $\mathrm{CNB}$ has been proven to be reliable and accurate for the diagnosis of both benign and malignant diseases of the breast. Imaging-pathology correlation is of critical importance in imaging-guided breast biopsies to detect possible sampling errors and avoid diagnostic delay. Several investigators have recommended re-biopsy or surgical excision in 9-18\% of cases [8, 10-12].

When microcalcifications detected in mammography are sampled, specimen radiography can ensure that sufficient amounts of microcalcification have been removed via cutting needle biopsy or vacuum biopsy; however, in US-guided biopsy, it is more difficult to demonstrate that the sample is sufficient $[13,14]$. US-guided biopsy is a technically easy procedure, and generally provides reliable sampling. However, sampling can be insufficient due to lesion size, morphological properties, inner structure, and location. In this study, the cases in which radiology-pathology discordance was found after US-guided cutting needle biopsy had heterogeneous lesions for which the assessment of sufficiency of sampling is not as easy as with biopsies taken for microcalcifications. US is used as the guide for all biopsies of lesions that are easily assessed sonographically. For percutaneous needle biopsies, US is a quick, practical, X-ray-free, cost effective, and reliable guide method which provides high patient comfort. With US, sampling is possible not only for solid masses but also for a broad range of lesion groups such as complex cysts, structural disruption, focal heterogeneity, and focal acoustic shadowing foci. However, especially in nonsolid masses and small lesions, demonstrating whether or not a sufficient sample has been obtained is not easy. If a lesion is easily seen on mammography, post-biopsy mammography control is capable of demonstrating that the sample has been obtained from the right region [13, 14]. However, in 14-gauge needle biopsies, changes are generally not sufficient to yield marked findings in mammography relating to the breast tissue and the non-calcified lesion. The diagnostic accuracy of imaging-guided CNB is quite high, and false negativity rates in the literature range from 0 to $9 \%$. However, when assessing biopsy results, possibility of false negativity should be considered. 
The frequency of imaging-histological discordance at percutaneous biopsy ranges from 1 to $6 \%$, and for lesions with discordant results at percutaneous biopsy subsequent surgical excision has demonstrated carcinoma in $0-64 \%$ of cases $[1,7,8]$. However, these data were obtained mainly from stereotactic biopsies. Liberman et al. $[1,15]$ reported that the frequency of imaging-histological discordance with US-guided percutaneous 14-gauge CNB was approximately $3 \%$, and the upgrade rate at re-biopsy was $10-25 \%$, in their large series. In the current study, the frequency of imaginghistological discordance and the upgrade rate at re-biopsy were $5.8 \%(58 / 989)$ and $27.5 \%(16 / 58)$, respectively, which is in agreement with the literature $[7,12]$.

In this study, the upgrade rate to carcinoma was $20 \%$ in BIRADS category 4 lesions and $53.8 \%$ in BI-RADS category 5 lesions.
This means that 1 in 2 BI-RADS 5 lesions and 1 in 5 BI-RADS 4 lesions in the histologically upgraded group showed malignancy in subsequent excisional biopsy.

In conclusion, we found imaging-histological discordance in $5.8 \%$ of lesions sampled with US-guided 14-gauge CNB. Imaginghistological discordance is an indication for surgical excision because of the high (27.5\%) prevalence of carcinoma in these lesions. Our study suggests that careful imaging-histological correlation can minimize the risk of delayed diagnosis of breast cancer.

\section{Disclosure Statement}

The authors declare that they have no conflict of interest.

\section{References}

1 Liberman L, Drotman MB, Morris EA, et al.: Imaginghistologic discordance at percutaneous breast biopsy. Cancer 2000;89:2538-2546.

2 Parikh J, Tickman R: Image-guided tissue sampling: where radiology meets pathology. Breast J 2005;11: 403-409.

3 American College of Radiology: Breast Imaging Reporting and Data System (BI-RADS), 4th ed. Reston, VA, ACR, 2003.

4 Cho SH, Park SH: Mimickers of breast malignancy on breast sonography. J Ultrasound Med 2013;32:2029_ 2036.

5 Liberman L: Percutaneous image-guided core breast biopsy. Radiol Clin North Am 2002;40:483-500.

6 Comstock CE: US-guided interventional procedures; in Feig SA (ed): 2005 Syllabus: Categorical Course in Diag nostic Radiology-Breast Imaging. Oak Brook, IL, Radiological Society of North America, 2005, pp. 155-168.
Meyer JE, Smith DN, Lester SC, et al.: Large-core needle biopsy: nonmalignant breast abnormalities evaluated with surgical excision or repeat core biopsy. Radiology 1998;206:717-720.

8 Youk JH, Kim EK, Kim MJ, et al.: Missed breast cancers at US-guided core needle biopsy: how to reduce them. Radiographics 2007;27:79-94.

9 Schueller G, Jaromi S, Ponhold L, et al.: US guided 14gauge core-needle breast biopsy: results of a validation study in 1,352 cases. Radiology 2008;248:406-413.

10 Dershaw DD, Morris EA, Liberman L, et al.: Nondiagnostic stereotaxic core breast biopsy: results of rebiopsy. Radiology 1996;198:323-325.

11 Whitman GJ, Erguvan-Dogan B, Yang WT, et al.: Ultrasound-guided breast biopsies. Ultrasound Clin 2006; 1:603-615.
12 Liberman L: Clinical management issues in percutaneous core breast biopsy. Radiol Clin North Am 2000;38: 791-807.

13 Liberman L, Evans WP 3rd, Dershaw DD, et al.: Radiography of microcalcifications in stereotaxic mammary core biopsy specimens. Radiology 1994;190:223-225.

14 Berg WA, Jaeger B, Campassi C, et al.: Predictive value of specimen radiography for core needle biopsy of noncalcified breast masses. AJR Am J Roentgenol 1998;171: 1671-1678.

15 Liberman L, Feng TL, Dershaw DD, et al.: Ultrasoundguided core breast biopsy: utility and cost-effectiveness. Radiology 1998;208:717-723. 